\title{
Flooding Speed in Wireless Multihop Networks with Randomized Beamforming
}

\author{
Vasil Mizorov $^{1}$, Jörg Widmer ${ }^{2}$, Robert Vilzmann ${ }^{3}$, and Petri Mähönen ${ }^{4}$ \\ 1 Siemens AG, Corporate Technology, D-81730 Munich, Germany \\ 2 DoCoMo Euro-Labs, D-80687 Munich, Germany \\ 3 Technische Universität München, D-80290 Munich, Germany \\ ${ }^{4}$ RWTH Aachen University, D-52072 Aachen, Germany
}

\begin{abstract}
This paper analyzes aspects of message propagation in multihop wireless networks with beamforming antennas. In particular, we focus our intention on the message propagation in the time domain. Our work uses a simulation based implementation of the 802.11 MAC protocol and a simplified version of a previously proposed MAC protocol, called BeamMAC [1]. Both protocols are compared under different network scenarios with several antenna array implementations (including an omnidirectional antenna).

Our conclusions confirm the advantages beamforming antennas have over omnidirectional antennas in wireless multihop networks. Reduced hop distances and reduced time for information dissemination speed up flooding of messages. Moreover, we observe the impact network topology parameters have on the overall performance of the message propagation.
\end{abstract}

Keywords: Ad Hoc Networks, antenna arrays, randomized beamforming, flooding speed.

\section{Introduction}

The concept of wireless ad hoc networking has been until recently considered only with omnidirectional antennas. Their advantage is that they are small, compact, spatial and radiate power omnidirectionally, i.e. equally in all spatial directions. However, they cause higher interference and block transmissions of other network nodes, significantly reducing the capacity and the throughput of the network. Seeking a way to increase the network capacity and throughput, directional (i.e. beamforming) antennas have been addressed. Their most important feature, to focus the energy into specific spatial directions, has proven to be appealing for providing higher network capacity and greater spatial reuse.

There are some downsides in implementing beamforming antennas for wireless ad hoc networking. Firstly, an antenna array means increased hardware size as opposed to the small size of the wireless gadgets. However, the latest technology allows antenna arrays to be smaller in size, making their implementation easier. Secondly, beamforming antennas must "know" the direction of the intended recipient. Otherwise, they might "miss" and radiate in a nonoptimal 
direction. Therefore, additional signal processing algorithms, like Direction-ofArrival (DoA) or Angle-of-Arrival (AoA) algorithms, are necessary for achieving optimal performance. Despite these facts, some research papers report achieving significantly larger gains in terms of network throughput when deploying beamforming antennas. However, they are based on knowing the neighbors' locations so each node can properly position its antenna beam (2], 4, 3]). The location information can be obtained by means of Global Positioning System (GPS) [6] or AoA or DoA estimation algorithms (4], [5]).

Bearing in mind battery life consumption, complexity and sometimes processing capabilities of the mobile devices, we believe that these algorithms will overburden the devices and reduce their usage time. Therefore, to reduce the implementation complexity and to simplify the communication, we use randomized beamforming [7. Nodes choose the direction of radiation randomly and avoid signal processing complexity. Thus, it turns out to be a practical approach when no a priori information is available about location of the nodes.

With respect to network topology properties, authors in [7] show that the randomized beamforming improves the connectivity in the network. Due to the longer links beamforming antennas provide, it is possible to "build a bridge" among previously isolated subnetworks [7. In addition, 9] discusses the hop distances when randomized beamforming is implemented. It shows that the network diameter, as well as the random node pair hop distance are significantly reduced in the case of randomized beamforming. These findings are very interesting considering that the randomized beamforming introduces zigzag paths, which may increase the hop distances and lead to slower message dissemination. However, authors in 7 and 9] do not consider the effects that a Medium Access Control (MAC) layer introduces to the process of message propagation.

The work presented in this paper aims to further investigate the communication features in wireless ad hoc networks with randomized beamforming. In particular, we focus our intention on the time domain of the message propagation in these networks. As this type of study was missing in the scope of the related papers [9] and [7, our work represents the first step towards more realistic approach in the investigation of the time-related features in the message propagation in networks implementing randomized beamforming. For comparison purposes, we simulate the IEEE $802.11 \mathrm{MAC}$ protocol with both, omnidirectional and beamforming antennas. We use flooding speed as main performance metric. In addition, we analyze the route discovery process and discuss its impact on the message propagation.

The remainder of this paper is organized as follows. Section 2 describes the proposed BeamMAC protocol. Section 3 explains the antenna and link model, as well as the scenarios used in our simulations. In section 4 we present the time aspects of the message propagation. In addition, we discuss the route discovery process and the impacts beamforming antennas have on it. Finally, Section 5 concludes the work. 


\section{Protocol Model}

Work done in the field of implementing beamforming antennas for ad hoc networking resulted in various modifications of the IEEE 802.11 MAC protocol. Some propose extending the Network Allocation Vector (NAV) into directional NAV (D-NAV) by keeping directionality related information [3]. Other proposals implement directional and omnidirectional transmission of Request-To-Send (RTS) and Clear-To-Send (CTS) messages [2]. However, as the 802.11 protocol was designed for omnidirectional transmissions, network performance can deteriorate due to issues specific to directional antennas [8].

Therefore, in order to investigate the time aspects of message propagation in wireless multihop networks, we evaluate a simplified version of the BeamMAC [1] protocol. It gains access to the wireless transmission channel using the following control packets:

- Announcement (ANN)

- Ready-To-Receive (RTR)

- Objection. (OBJ)

A node willing to initiate a data transmission, must announce it beforehand. For this purpose, it sends an ANN to inform the transmitter's surrounding of the forthcoming transmission. In other words, each desired transmission is "simulated" before being carried out. If the intended destination of the communication, for which the ANN packet is meant, is idle (i.e. not transmitting or receiving), it transmits an RTR packet back to the transmitter. The idea here is to inform the transmitter that the desired addressee is available. Upon reception of an ANN, each neighbor currently engaged in a parallel communication as a receiver, determines the interference that would be caused by the forthcoming transmission.

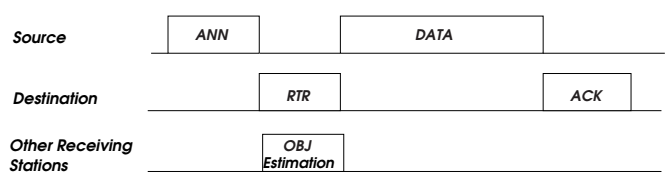

Fig. 1. BeamMAC Channel Access

If the interference is so high to degrade the ongoing communication, the receiver sends an OBJ back to the sender of the ANN. In case the level of the interference maintains an acceptable level, the receiver does not send an OBJ back. In case an OBJ is received, the node enters a backoff state. Details of how an ANN packet is assessed by a receiving node are not discussed in this paper. Instead, we refer the interested reader to [1]. When the transmission is successfully "simulated" (ANN and RTR are sent, and no OBJ is received), the actual data packet can be sent. Upon error-free reception of the data packet, the receiving node transmits an acknowledgment (ACK) back. 


\section{Network Model}

\subsection{Antenna Model}

Antenna arrays, used in Multiple Input Multiple Output (MIMO) systems to increase the user data rate, can have different shapes; most prominently, linear or circular. Antennas with linear geometry are referred to as linear antenna array, whereas antennas with circular geometry are known as circular antenna arrays. Circular antenna arrays offer higher diversity and improved link capacity ([17], [18. Thus, the antenna model used in our simulations is Uniform Circular Array (UCA) [7]. An UCA array comprises $m$ identical isotropic radiators placed uniformly on a closed circumference. Each antenna element transmits with the same power $p_{t} / m$ at a wavelength $\lambda=\frac{c}{f}$, with $c=3 \cdot 10^{8} \mathrm{~m} / \mathrm{s}$ and carrier frequency $f$. By implementing a phase shift between the array elements, the resulting antenna beam pattern can be controlled. The shape of the resulting beam depends on the target direction $\Theta_{b}$, known as boresight direction, and the number of antenna elements. Examples of antenna patterns for UCA antenna with $m=4$ elements are shown in Figure2.

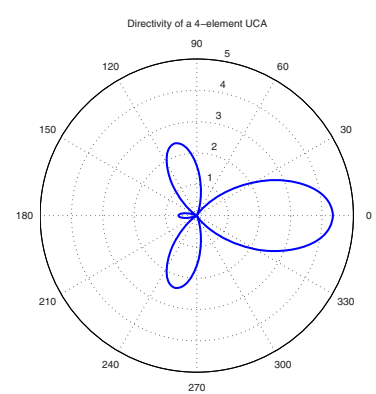

(a) $\Theta_{b}=0^{\circ}$

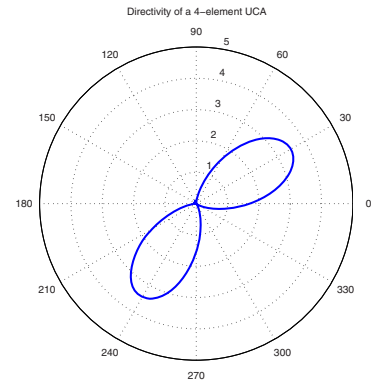

(b) $\Theta_{b}=30^{\circ}$

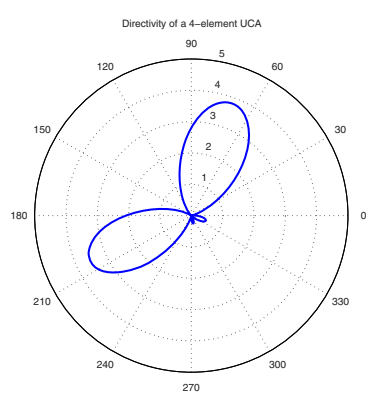

(c) $\Theta_{b}=70^{\circ}$

Fig. 2. Gain patterns of UCA with $m=4$ elements

In general, an antenna pattern consists of a main lobe and side lobes. The main lobe represents the radiation in the desired direction, whereas the side lobes refer to the radiation in all other directions. It can be noted from antenna theory [10 that with an increase in the number of elements in the antenna array the radiated power in the direction of the main lobe increases. Note that due to antenna reciprocity, the gain characteristic is valid for both, transmission and reception.

\subsection{Wireless Link Model}

The wireless link model is based on a line-of-sight communication between two nodes, given their transmission parameters and their distance. Figure 3 depicts the implemented link model. 


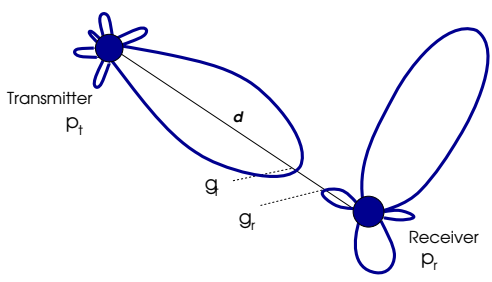

Fig. 3. Wireless link model

One node transmits the signal with power $p_{t}$, which is received by the other node with power $p_{r}$. The gain of the antenna at the transmitting node is $g_{t}$. The gain of the receiver's antenna in the corresponding direction toward the transmitter is $g_{r}$. Thus, we can write

$$
\frac{p_{r}}{p_{t}}=g_{t} g_{r}\left(\frac{d}{1 m}\right)^{-\alpha},
$$

where $\alpha$ represents the pathloss exponent of the propagation environment. The value of $\alpha$ is environment-dependent and is approximately $\alpha=2$ for a free space scenario and $\alpha=3 \ldots 5$ for urban areas [10]. The link establishment between two nodes assumes that the received power $p_{r}$ is above the receiver sensitivity $p_{r 0}$, that is

$$
p_{r} \geq p_{\text {ro }} .
$$

In the following, we assume that all nodes have the same transmission power $p_{t}$ and reception sensitivity $p_{\text {ro }}$. Thus, considering the fact that antenna pattern reciprocity holds (same antenna pattern for transmission and reception), all links in the network can be considered as bidirectional (or undirected) links. That is, if a node $\mathrm{A}$ can communicate with node $\mathrm{B}$, then node $\mathrm{B}$ can communicate with node A, as well. One should note that our simulation model does not implement propagation phenomena like fading.

\subsection{Randomized Beamforming}

As mentioned in Section 1, in order to avoid implementing complex signal processing algorithms, we use a communication paradigm referred to as randomized beamforming [7. Its implementation is based on nodes choosing a random direction where to point their antenna beams. With choosing both, the boresight direction and the antenna array direction, uniformly distributed in the interval $[0,2 \pi]$ the shape of the resulting pattern is fully described. In addition, all nodes keep their beam direction constant the whole time, i.e. once chosen it does not change.

\subsection{Network Topology and Scenarios}

The network topology in our simulations comprise $n$ nodes distributed uniformly at random on a square area with side length $l$. For obtaining the node coordinates 
(x, y) we use the Mersenne Twister pseudo-random number generator [1]. Nodes in the network are static. We use a simple flooding mechanism to disseminate messages into the network. One node sends a packet and all other nodes forward the packet until received by every node.

To more closely model reality, we borrow parameters from the IEEE 802.11 standard [12. Namely, parameters used in our simulations are: frequency $f=2 \mathrm{GHz}$, pathloss exponent $\alpha=3$ (urban area), maximum transmission power $p_{t}=0.1 \mathrm{~W}$, communication threshold $P_{r 0}=-111 \mathrm{~dB}$, sensitivity threshold (for 802.11) $P_{s 0}=-121 \mathrm{~dB}$ and transmission range for omnidirectional antenna $T_{x}$-Range $=$ $121 \mathrm{~m}$. In addition, we simulate the 802.11 protocol only with omnidirectional antennas, whereas the BeamMAC protocol with both, omnidirectional and UCA antennas (particularly UCA with $m=4$ or UCA 4 and $m=10$ or UCA10).

Table 1. Network scenarios

\begin{tabular}{cccc}
\hline Network size & $n$ & $l(m)$ & Area $\left(\mathrm{km}^{2}\right)$ \\
\hline Small & 100 & 577 & 0.33 \\
Medium & 500 & 1290 & 1.66 \\
Large & 2000 & 2580 & 6.65 \\
\hline
\end{tabular}

We conduct our simulations with node density $n / l^{2}=300 \mathrm{~km}^{-2}$. In order to obtain at least $95 \%$ connectivity in the network, we use calculations taken from [13] and calculate the area for a certain number of nodes so to guarantee the required connectivity. In fact, the connectivity in the network is above $99 \%$. In order to perform a thorough analysis of the message dissemination process, we regard three scenarios in our simulations [15. Namely, we use different network sizes, i.e. small, medium and large, as we want to clearly investigate any connection between the size of the network and the performance of the both protocols. The number of nodes for each network scenario is 100, 500 and 2000 nodes, respectively. In addition, the network area is $0.33 \mathrm{~km}^{2}, 1.66 \mathrm{~km}^{2}$ and 6.65 $\mathrm{km}^{2}$, respectively. The simulated scenarios (number of nodes, length, network area) are given in Table 1

\section{Simulation Analysis}

For the purpose of conducting the analysis we apply a protocol driven ad hoc network simulation tool (PANTS). It is an event-based simulator developed in $\mathrm{C}++$ language, incorporating realistic models for beamforming antenna patterns, calculated using accurate formulas provided by antenna theory [10] and moreover, implementing the two investigated protocols, IEEE 802.11 and BeamMAC. For visualization purposes of the network topology and the distribution of the nodes in it, our simulation tool uses the Library of Efficient Data Types and Algorithms (LEDA) [14. 
With respect to the simulated parameters, we were interested in the flooding speed, flooding time and route reply time. The first one describes the speed with which a message propagates in the network, in terms of how many nodes have received the message until a certain time instant. The second parameter tells us how long it takes to flood a message in the network. Finally, the third parameter helps us better understand the route reply in networks implementing randomized beamforming.

To be compliant with the protocol specifications, for 802.11 we implement broadcasting as defined in the IEEE 802.11 standard [12] (without RTS/CTS control handshake) and we omit the ACK packet in BeamMAC implementation. In addition, in the route reply process we make use of the 802.11 DCF function (RTS/CTS scheme) and all of the BeamMAC protocol functions.

For accuracy purposes, we run a large number of simulations for every scenario. In particular, for the small and medium network we use 200 runs and for the large network we use 100 runs (due to large memory consumption). In addition, in our graphs we give a confidence interval of one sigma ( $\sigma$ being standard deviation) which equals to a confidence level of $68.27 \%$.

\subsection{Flooding Speed}

We define the flooding speed as a curve that gives the percentage of flooded nodes depending on the time. We calculate the number of flooded nodes on every packet transmission and the result (nodes, time) is represented as a point of the flooding speed curve. Figures 46 depict the results we obtained from the considered scenarios given in Table 1.

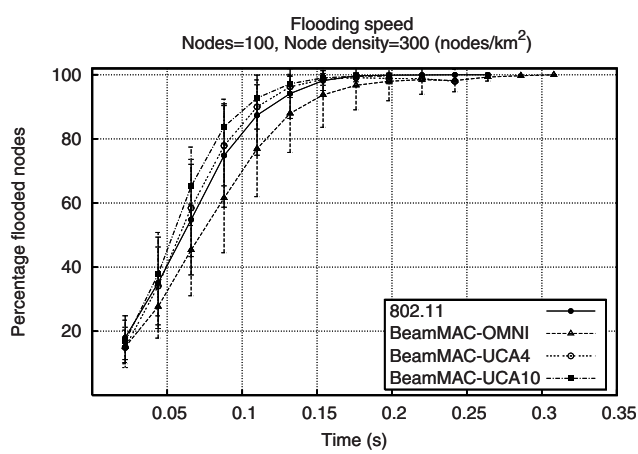

Fig. 4. Flooding speed for 802.11 and BeamMAC protocol, small network

Figure 4 gives the results for the small network scenario $(n=100)$. It compares several different combinations of antenna and MAC protocol, namely the 802.11 protocol (implemented with OMNI antenna) and the BeamMAC protocol implemented with directional antenna (UCA4 and UCA10) and OMNI antenna. The obtained flooding speed curves are close to one another, which means that the advantages of the beamforming antennas are hardly noticeable. 
There is a minor difference in the Percentage of Flooded Nodes (PFN) between the BeamMAC and the 802.11 for a fixed value of the time, which is about 1\%-5\% for BeamMAC-UCA4 and BeamMAC-UCA10, respectively. However, the BeamMAC-UCA10 scheme has the highest flooding speed. The poor performance observed in the small network scenario is due to the impact of the border effects on the message propagation. Namely, caused by the random beamforming and the fact that the network area is relatively small, many nodes transmit outside the area. This is more visible in the beamforming case, as their transmission range is up to several times bigger than the one of the omnidirectional antennas.

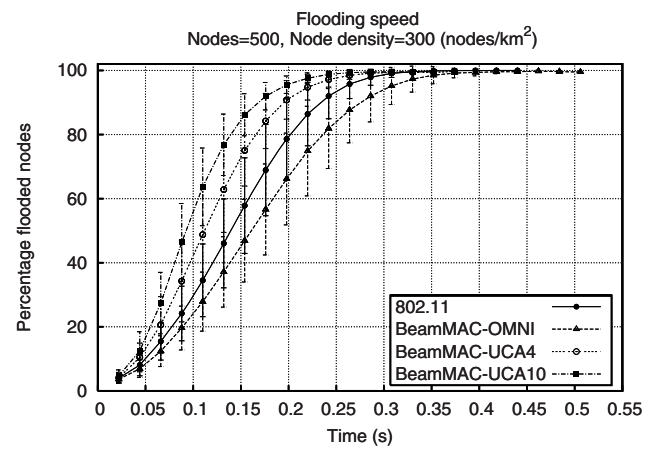

Fig. 5. Flooding speed for 802.11 and BeamMAC protocol, medium network

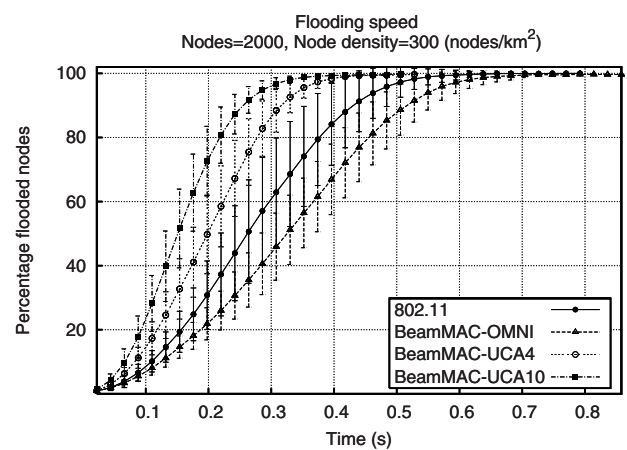

Fig. 6. Flooding speed for 802.11 and BeamMAC protocol, large network

The medium network scenario shown on Figure 5 as well as the large network scenario on Figure 6 show an improvement in the message propagation for beamforming antennas. In particular, the larger the network size is, the better the flooding speed of the beamforming antennas. In the latter network, the difference between the BeamMAC-UCA4 and the 802.11 is more than 20\%, which makes the UCA4 antenna a more sensible and more practical solution. The impact that border effects have on the message propagation is minor, as a larger portion of the nodes radiate inside the network area and less transmissions are void. 
It can be noted from Figures 46 that the performance of the combination of the BeamMAC protocol and omnidirectional antenna (BeamMAC-OMNI) experiences the poorest performance. The main reason for this inferior behavior is protocol related. Namely, the BeamMAC protocol takes longer to access the wireless channel (ANN and RTR) compared with the 802.11 protocol (only DIFS - Distributed Inter Frame Space). Consequently, this has proven to be reason for the slower flooding speed of the BeamMAC-OMNI implementation.

\subsection{Flooding Time}

The flooding time parameter is defined as time it takes to flood the whole network. It can be derived from the flooding speed curves, as the time instance when the percentage of flooded nodes is 100 (i.e. whole network is flooded). We show the obtained values from our simulations in Figure 7 . The parameter flooding time can be understood as a follow-up to the flooding speed analysis. In the small network scenario there is scarcely a difference between the omnidirectional and directional antenna implementation. However, increasing the network size the difference in the flooding time between the directional and omnidirectional antenna implementation is continuously reducing, meaning that beamforming antennas have the dominance in larger network areas. The reduction of the flooding time is about 20\%-30\% (large network scenario) for the BeamMAC-UCA4 and for the BeamMAC-UCA10, respectively.

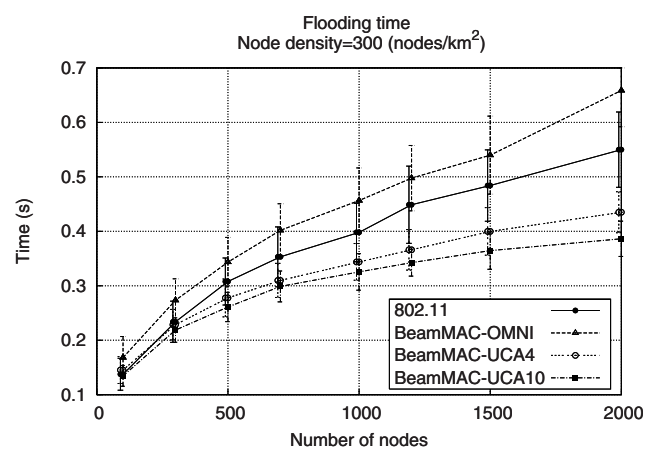

Fig. 7. Flooding time for 802.11 and BeamMAC protocol

\subsection{Route Reply (RREP) Time}

In addition to the flooding mechanism analyzed in Section 4.1, we implemented and analyzed a route reply mechanism, as well. We believe that this parameter will give us more understanding about the time it takes two nodes to establish a path.

The initiator of the route discovery (i.e. the source) broadcasts a packet to the destination, which after receiving it, generates and sends a route reply packet to the source. The route reply packet is not broadcasted, but rather sent using a 
hop-by-hop unicasting. The route reply mechanism is based on a source routing concept, i.e. the reply packet follows the same path on which the route request came from.

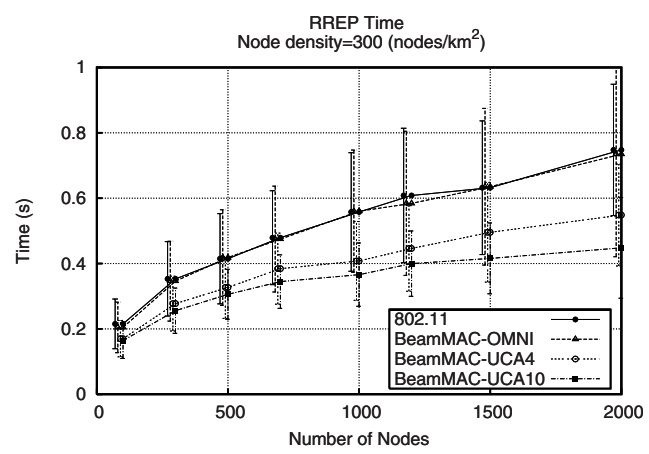

Fig. 8. Route reply time for 802.11 and BeamMAC protocol

As Figure 8 shows, the network area has a negative impact on the route discovery process, as well. On the one hand, considering the small network scenario, we confirm that there is an insignificant reduction of the route reply time with beamforming antennas. On the other hand, considering the large network scenario, we notice a superior performance of the BeamMAC protocol. The route reply time has been reduced by about 30\%-40\% in the case of the BeamMACUCA4 and the BeamMAC-UCA10, respectively. Due to the previous analysis of the flooding speed, we can see here that having faster message propagation ensures quicker delivery to the desired destination. This in turn, helps in achieving better route reply times.

\section{Conclusion}

Our work outlines the positive impact that the beamforming antennas have on the information dissemination in wireless multihop networks. Our study is performed by simulating realistic network scenarios, considering the very popular 802.11 MAC protocol and a simplified version of the BeamMAC protocol. In addition, we use parameters adopted directly from the IEEE 802.11 standard 12. Results presented in this paper represent a performance comparison of the broadcast mechanism in multihop networks. Together with the findings in 7 ] and [9], we provide a thorough analysis of the parameters related to the network topology as well as to the message dissemination in these networks.

Although our approach includes a simple flooding model and a simple sourcerouting based route discovery process, we show that the beamforming antennas outperform the omnidirectional antennas. They provide faster message dissemination and faster route discovery process. In addition, we identify their downsides (e.g. void transmission) when implemented in small network scenarios. However, 
in large network area scenarios the beamforming antennas have proven to be superior. This approach could be the worst case analysis when there is no other network information available. As soon as more topology related information is available, nodes can use sophisticated location finding algorithms to adapt or optimize their radiation direction. Moreover, this particular study can be of further help in service discovery scenarios in wireless multihop networks. By simply defining a threshold when the service announcement or service query is successful (e.g. $80 \%$ of nodes receive the packet), looking into Figures 4 [6] we can obtain the time it takes for a certain service to be properly advertised.

We strongly believe that the implementation of beamforming antennas has an enormous potential in wireless multihop networks. Therefore, more in-depth analysis is required which will investigate the actual routing and will look into issues related to cross-layer optimization.

\section{Acknowledgment}

The authors would like to thank Imad Aad from DoCoMo Euro-Labs and Christian Bettstetter (previously with DoCoMo, now with University of Klagenfurt) for various discussions and very useful comments.

\section{References}

1. R. Vilzmann, C. Bettstetter, C. Hartmann: BeamMAC: A New Paradigm for Medium Access in Wireless Networks, International Journal of Electronics and Communications (AEÜ), Volume 60, Number 1, (Jan. 2006) 3-7.

2. M. Takai, J. Martin, A. Ren, R. Bagrodia: Directional Virtual Carrier Sensing for Directional Antennas in Mobile Ad Hoc Networks, In Proc. 3rd ACM MobiHoc, Switzerland, (June 2002) 183-193.

3. R. Roy Choudhury, N. H. Vaidya: Ad Hoc Routing Using Directional Antennas, Technical Report, Coordinated Science Laboratary, University of Illinois at Urbana-Champaign (Aug. 2002).

4. H. Singh, S. Singh: A MAC Protocol based on Adaptive Beamforming for Ad Hoc Networks, In Proc. IEEE PIMRC, China, (Sept. 2003) 1346-1350.

5. H. Singh, S. Singh: Smart-802.11b MAC Protocol for Use with Smart Antennas, In Proc. IEEE ICC, France, Volume 6, (June 2004) 3684-3688.

6. Y.-B. Ko, V. Shankarkumar, N. H. Vaidya: Medium Access Control Protocols using Directional Antennas in Ad hoc Networks, In Proc. IEEE Infocom, Israel, (March 2000), 13-21.

7. C. Bettstetter, C. Hartmann, C. Moser: How Does Randomized Beamforming Improve the Connectivity of Ad Hoc Networks?, In Proc. IEEE ICC, Korea, (May 2005), 3380-3385.

8. R. Vilzmann, C. Bettstetter: A Survey on MAC Protocols for Ad Hoc Networks with Directional Antennas, In Proc. EUNICE Open European Summer School, Spain, (July 2005), 268-274.

9. R. Vilzmann, C. Bettstetter, D. Medina, C. Hartmann: Hop Distances and Flooding in Wireless Multihop Networks with Randomized Beamforming, In Proc. ACM MSWIM, Canada, (Oct. 2005), 20-27. 
10. Constantine A. Balanis: Antenna Theory, Analysis and Design, John Wiley \& Sons, Inc., 2nd Edition, (1997).

11. M. Matsumoto, T. Nishimura: Mersenne Twister: A 623-Dimensionally Equidistributed Uniform Pseudo-Random Number Generator, ACM Transactions on Modeling and Computer Simulation, Volume 8, Number 1, (1998), 3-30.

12. IEEE Standards Board: Part 11: Wireless LAN Medium Access Control (MAC) and Physical Layer (PHY) Specifications, (1999), http://www.ieee802.org/11/.

13. C. Bettstetter: Mobility Modelling, Connectivity, and Adaptive Clustering in Ad Hoc Networks, PhD Thesis, Technische Universität München, Germany, (Oct. 2003).

14. Algorithmic Solutions: LEDA - Library of Efficient Data Types and Algorithms, (Dec. 2005), http://www.algorithmic-solutions.com/enleda.htm.

15. V. Mizorov: Routing in Ad Hoc Networks with Beamforming Antennas, Master Thesis, RWTH Aachen University, (Dec. 2005).

16. T. Korakis, G. Jakllari, L. Tassiulas: A MAC protocol for full exploitation of directional antennas in ad hoc wireless networks, In Proc. 4th ACM MobiHoc, USA, (June 2003), 98-107.

17. W. Weichselberger, G. L. de Echazarreta: Comparison of Circular and Linear Antenna Arrays with respect to the UMTS Link Level, COST 260 Management Committee and Working Groups Meeting, Göteburg, Sweden, (May 2001).

18. N. Razavi-Ghods, M. Abdalla, S. Salous: Characterisation of MIMO Propagation Channels Using Directional Antenna Arrays, in Proc. IEE International Conference on 3G Mobile Communication Technologies (3G2004), London, UK, (October 2004). 